\title{
Finite $\beta$ Effects on Short Wavelength Ion Temperature Gradient Modes
}

\author{
M. Jagannath ${ }^{1}$ : J. Chowdhury ${ }^{2}$, R. Ganesh ${ }^{1}$, and L. Villard ${ }^{3}$ \\ ${ }^{1}$ Institute for Plasma Research, Bhat, Gandhinagar, 382428, India \\ ${ }^{2}$ University of Warwick, Coventry CV4 7AL, UK and \\ ${ }^{3}$ Swiss Plasma Center, EPFL, 1015 Lausanne, Switzerland
}

\begin{abstract}
The electromagnetic effect is studied on the short wavelength branch of the ion temperature gradient mode in the linear regime for the first time using a global gyrokinetic model. The short wavelength ion temperature gradient mode growth rate is found to be reduced in the presence of electromagnetic perturbations at finite plasma $\beta$. The effect on real frequency is found to be weak. The threshold value of $\eta_{i}$ is found to increase for the mode as the magnitude of $\beta$ is increased. The global mode structure of the short wavelength branch of the ion temperature gradient mode is compared with the conventional branch. The magnetic character of the mode, measured as the ratio of mode average square values of electromagnetic potential to electrostatic potential, is found to increase with increasing values of the plasma $\beta$. The mixing length estimate for flux shows that the maximum contribution still comes from the long wavelengths modes. The magnitude of the flux decreases with increasing $\beta$.
\end{abstract}

\footnotetext{
* jaga.physics94@gmail.com; jagannath.mahapatra@ipr.res.in
} 


\section{INTRODUCTION}

It is now well known that magnetically confined plasmas bear the prospects of a future fusion reactor. The progress in this endeavor, however, is hamstrung by technological and physics issues. With the available technology, the success of such a goal depends on understanding the nature of the instabilities and controlling them. The Larmor radius scale instabilities, which are also called microinstabilities, cause anomalous transport leading to expulsion of heat and particles from the system. This is undesirable and has to be avoided or at least reduced. These microinstabilities feed on the free energy available in the inhomogeneities in temperature and density of the particles. The ion temperature gradient (ITG) [1 5] mode, trapped electron mode (TEM) [6-11], and universal drift instabilities [1214], are some of the examples of such unstable modes at the ion scale while the electron temperature gradient mode (ETG) [15-18] is another class of instabilities at the electron scale. Finite $\beta$ plasmas also give rise to electromagnetic instabilities such as microtearing modes (MTM) [19 27] and kinetic ballooning modes (KBM) [28 31]. Intermediate to these scales, there exists a class of instabilities driven by a strong ion temperature gradient. This mode occurs on the shorter scale than the conventional ITG mode mentioned above and therefore, is called the short wavelength ion temperature gradient (SWITG) mode [32 40]. Usually, the ion temperature gradient driven mode exists for $k_{\perp} \rho_{L i} \leq 1$. However, when the gradient scale lengths are very short, one can observe another branch of ITG mode which becomes unstable at scales $k_{\perp} \rho_{L i}>1$.

With the progress in the tokamak fusion research, tokamaks are now able to operate in advanced scenarios. Some of such scenarios offer very strong gradients. For example in the region of internal transport barriers, the gradients can be very strong [41]. In H mode plasma as well, the profiles turn very steep, resulting in very strong gradients [42]. Apart from other modes, the shorter wavelength mode might be very unstable with the $k$ spectrum extending well beyond the conventional modes [43] for parameters relevant to tokamak experiments.

The current and future tokamak machines confine plasma at a higher pressure which is expressed in $\beta$. Therefore, the modification of the mode properties in the presence of finite $\beta$ has to be taken into consideration. The $\beta$ effect turns out to be very important for both inherently electrostatic and electromagnetic modes. While the electromagnetic instabilities arise and become stronger with increasing $\beta$, the same finite- $\beta$ effect suppresses 
the electrostatic instabilities. In the presence of fast ion, high $\beta$ leads to the reduction in the profile stiffness observed in many experiments and confirmed in subsequent gyrokinetic studies [44]. The role of electromagnetic perturbations on drift modes has been studied extensively. While the electromagnetic perturbation is observed to give rise to instabilities such as the kinetic ballooning mode (KBM) [28 31], tearing and microtearing modes [1926], etc., the same is found to stabilize some other drift modes such as the ITG mode, trapped electron mode, universal drift modes, etc [13, 45,48]. The stabilizing effect of the electromagnetic perturbation on the ITG mode can be attributed to the field line bending induced by the electromagnetic perturbations. Although electromagnetic effects on ITG mode are well known, the effect of the electromagnetic perturbation on the SWITG mode is not known so far in detail [37]. Previously known finite $\beta$ studies on the SWITG mode are in slab geometry [36] and in the local limit [37]. We know that the SWITG mode is inherently an ion mode [37, and the magnetic shear has a stabilizing effect [36]. The dependence of the mode on $T_{e} / T_{i}$, toroidicity, magnetic shear, safety factor, etc., are studied in Refs [38, 39]. The role of $E \times B$ shear has been studied in Ref. [36]. Thus the properties of the SWITG mode have been extensively studied in the electrostatic limit. However, in the presence of $\beta$ or electromagnetic perturbation, which corresponds to a more realistic scenario of tokamak experiments, the detail properties of the SWITG mode have not been investigated so far using a global model. To close this gap in the knowledge of SWITG, in the present work, we explore the properties of the SWITG mode in the presence of finite $\beta$. For this reason, we use the electromagnetic version EM-GLOGYSTO [31, 49, 50] of the global gyrokinetic linear model GLOGYSTO [51 53]. This code has been extensively used to study electrostatic and electromagnetic modes. For example, in the electrostatic limit the code is used to study ITG [4, 52, TEM [10], SWITG [39] modes while in the electromagnetic limit KBM [31, 49, 50], and MTM [24, 54] modes are studied in detail. The present

manuscript is arranged as follows. Section II describes the model briefly. In Section III, the profiles and parameters used in the present simulations, results observed in the presence of finite $\beta$ are described. Finally, the results are summarized in Section IV.

\section{THE SIMULATION MODEL}

The EM-GLOGYSTO code is a global spectral code that calculates the real frequency and growth rates of unstable modes for a given equilibrium using the Nyquist method and also 
gives the eigenmode structure. Both ion and electron species are considered fully gyrokinetic. The equilibrium considered is circular and axisymmetric and can include the Shafranov shift. The code can treat trapped and passing particles separately and calculates the finite Larmor radius (FLR) effect to all orders. It includes all kinetic effects including Landau damping physics. The model includes both transverse and compressional perturbation in the electromagnetic limit. In the present study, however, we consider $\phi$ and $A_{\|}$components only. Before proceeding to present the results we briefly summarize the simulation model in this section. A greater detail of the model can be found in Refs. [51, 52]. For electromagnetic calculations, readers are referred to Refs. [31, 49]. The perturbed density for a species $j$ can be expressed as the sum of adiabatic and nonadiabatic parts as follows.

$$
\tilde{n}_{j}(\mathbf{r} ; \omega)=-\left(\frac{q_{j} N}{T_{j}}\right)\left[\tilde{\varphi}+\int d \mathbf{k} \exp (i \mathbf{k} \cdot \mathbf{r}) \int d \mathbf{v} \frac{f_{M j}}{N}\left(\omega-\omega_{j}^{*}\right)\left(i \mathcal{U}_{j}\right) \tilde{\varphi}(\mathbf{k} ; \omega) J_{0}^{2}\left(x_{L j}\right)\right]
$$

In the above equation $q_{j}$ and $T_{j}$ are the charge and temperature for the species $j, \mathrm{~N}$ stands for the equilibrium density, $\tilde{\varphi}$ is the perturbed electrostatic potential, $\mathbf{k}=\kappa \vec{e}_{r}+k_{\theta} \vec{e}_{\theta}+k_{\phi} \vec{e}_{\phi}$ where radial wave vector $\kappa=(2 \pi / \Delta r) k_{r}$, with $\Delta r=r_{u}-r_{l}$ defines the radial domain, poloidal wave vector $k_{\theta}=m / r$, toroidal wave vector $k_{\phi}=n / R$; and $\left(k_{r}, m, n\right)$ are radial, poloidal and toroidal mode numbers respectively. This model uses a toroidal coordinate system with $(r, \theta, \phi)$ representing radial, poloidal, and toroidal coordinates respectively. Similarly, $\left(\kappa, k_{\theta}, k_{\phi}\right)$ represent their respective conjugate coordinates in Fourier space. The diamagnetic drift frequency is given by $\omega_{j}^{*}=\omega_{n j}\left[1+\frac{\eta_{j}}{2}\left(\frac{v^{2}}{v_{t h j}^{2}}-3\right)\right]$ where $\omega_{n j}=\left(T_{j} \nabla_{n} \ln N k_{\theta}\right) /\left(q_{j} B\right), \nabla_{n}=-r B_{p} \frac{\partial}{\partial \psi}$, and $\eta_{j}=\left(d \ln T_{j}\right) /(d \ln N), v_{t h j}$ is the thermal velocity of species $j$. The Bessel function $J_{0}\left(x_{L j}\right)$ with $x_{L j}=k_{\perp} \rho_{L j}$, incorporates the full finite Larmor radius effect. Note that here $m$ and $n$ are poloidal and toroidal wave numbers, $q(s)$ is the safety factor, $k_{\theta}$ is the poloidal wave vector, $B_{p}$ is the poloidal magnetic field. $f_{M j}$ is a local Maxwellian for species $j$ of mass $m_{j}$ and is given by

$$
f_{M j}\left(\varepsilon_{j}, \psi\right)=\frac{N(\psi)}{\left(\frac{2 \pi T_{j}(\psi)}{m_{j}}\right)^{3 / 2}} \exp \left(-\frac{\frac{1}{2} m_{j} v^{2}}{T_{j}(\psi)}\right)
$$


The term $\mathcal{U}_{j}$ represents the guiding center propagator for the passing particles where,

$$
i \mathcal{U}_{j}=\sum_{p, p^{\prime}} \frac{J_{p}\left(x_{t j}^{\sigma}\right) J_{p^{\prime}}\left(x_{t j}^{\sigma}\right)}{\omega-\sigma k_{\|}\left|v_{\|}\right|-p \omega_{t}} \exp \left(i\left(p-p^{\prime}\right)\left(\theta-\bar{\theta}_{\sigma}\right)\right)
$$

with $x_{t j}^{\sigma}=k_{\perp} \xi_{j}^{\sigma}, \xi_{j}^{\sigma}=v_{d j} / \omega_{t}, v_{d j}=\left(v_{\perp}^{2} / 2+v_{\|}^{2}\right) /\left(\omega_{c j} R\right), \mu=\frac{v_{\perp}^{2}}{2 B}, \omega_{t}=\sigma\left|v_{\|}\right| /(q(s) R), \sigma=$ \pm 1 ( $\operatorname{sign}$ of $\left.v_{\|}\right), \varepsilon_{j}=\frac{1}{2} m_{j} v^{2}$. The perpendicular and parallel wave-vectors are given as $k_{\perp}=\sqrt{\kappa^{2}+k_{\theta}^{2}}, k_{\|}=[n q(s)-m] /(q(s) R)$. Also note that $\bar{\theta}$ is given by $\tan \bar{\theta}=-\kappa / k_{\theta}$. The Bessel functions contain the effect of $\nabla \mathrm{B}$ and curvature drifts through arguments $\left(x_{t j}^{\sigma}=\right.$ $k_{\perp} v_{d j}\left(\omega_{t}\right)$. Thus the Bessel functions in Eq. (2) take in to effect the coupling of the flux surfaces and also the coupling of the neighboring poloidal modes. Note that the argument of the Bessel functions $J_{p}$ in Eq.(2), $x_{t j}^{\sigma}=k_{\perp} \xi_{j}^{\sigma}$ also takes into account the effect of transit frequency $\omega_{t}$. The quasi-neutrality condition then gives

$$
\sum_{j} \tilde{n}_{j}(\mathbf{r} ; \omega)=0
$$

In the case of electrostatic fluctuations only, this leads to an eigenvalue problem, with $\omega$ and $\tilde{\varphi}$ being eigenvalues and eigenvectors and can be solved in Fourier space. For fully gyrokinetic ions and electrons with only passing particles we have:

$$
\sum_{\mathbf{k}^{\prime}} \sum_{j=\mathrm{i}, \mathrm{e}} \hat{\mathcal{M}}_{\mathbf{k}, \mathbf{k}^{\prime}}^{j} \tilde{\varphi}_{\mathbf{k}^{\prime}}=0
$$

For an axisymmetric system, one can fix the toroidal mode number $n$, and thus $\mathbf{k}=(\kappa, m)$ for the wave vector represents the radial and poloidal wave numbers $\kappa$ and $m$, respectively. Hence, $\mathbf{k}=(\kappa, m)$ and $\mathbf{k}^{\prime}=\left(\kappa^{\prime}, m^{\prime}\right)$. Here, $m^{\prime}=n q\left(s_{0}\right) \pm \delta m$, where $q\left(s_{0}\right)$ is the q-value at $s=s_{0}$ and $\delta m$ decides the range of poloidal mode number. With the inclusion of the electromagnetic perturbations, but neglecting $\delta B_{\|}$, Eq.(1) above is modified as [31, 49] $\tilde{n}_{j}(\mathbf{r} ; \omega)=-\left(\frac{q_{j} N}{T_{j}}\right)\left[\tilde{\varphi}+\int d \mathbf{k} \exp (i \mathbf{k} \cdot \mathbf{r}) \int d \mathbf{v} \frac{f_{M j}}{N}\left(\omega-\omega_{j}^{*}\right)\left(i \mathcal{U}_{j}\right)\left[\tilde{\varphi}(\mathbf{k} ; \omega)-v_{\|} \tilde{A}_{\|}(\mathbf{k} ; \omega)\right] J_{0}^{2}\left(x_{L j}\right)\right]$ 
where $\tilde{A}_{\|}$is the parallel component of the vector potential. The perturbed parallel current density can be written as,

$\tilde{j}_{\| j}(\mathbf{r} ; \omega)=-\left(\frac{q_{j}^{2}}{T_{j}}\right)\left[\int d \mathbf{k} \exp (i \mathbf{k} \cdot \mathbf{r}) \int v_{\|} d \mathbf{v} f_{M j}\left(\omega-\omega_{j}^{*}\right)\left(i \mathcal{U}_{j}\right)\left[\tilde{\varphi}(\mathbf{k} ;)-v_{\|} \tilde{A}_{\|}(\mathbf{k} ;)\right] J_{0}^{2}\left(x_{L j}\right)\right]$

With the quasi-neutrality condition Eq.(3), and Ampere's law

$$
\frac{1}{\mu_{0}} \nabla_{\perp}^{2} \tilde{A}_{\|}=-\sum_{j} \tilde{j_{\| j}}
$$

We finally arrive at a linear system of equations as follows

$$
\sum_{\mathbf{k}^{\prime}} \sum_{j=\mathrm{i}, \mathbf{e}} \hat{\mathcal{M}}_{\mathbf{k}, \mathbf{k}^{\prime}}^{j}\left(\begin{array}{c}
\tilde{\varphi}_{\mathbf{k}^{\prime}} \\
\tilde{A}_{\| \mathbf{k}^{\prime}}
\end{array}\right)=0
$$

This forms the core of the simulation model used in the present study. Note that Eq.(5) above represents a complex eigenvalue equation for the (complex) eigen frequency $\omega$, which is found numerically based on the Nyquist theorem. More details can be found in Ref. [53]. Moreover, a detail description of the set of equation included in Eq.(5) has been given in the Appendix. The geometry considered is circular, large aspect ratio, axisymmetric and unshifted. This code EM-GLOGYSTO is widely used to study many of the electrostatic and electromagnetic modes, such as the ITG [4, 52, TEM [10], universal toroidal mode [13], etc, in the electrostatic limit, and KBM [31, 49, 50], and MTM [24, 54] modes in the electromagnetic limit. This code is capable of studying modes in the shorter wavelength as well and was already used to study the SWITG [39] with trapped electrons. 


\section{RESULTS AND DISCUSSION}

\section{Parameters and Profiles}

For the present study, we consider the following profiles and parameters.

Parameters:

- B-field : $B_{0}=1.0$ Tesla, $\frac{m_{i}}{m_{e}}=1836$

- Temperature : $T_{0}=T\left(s_{0}\right)=7.5 \mathrm{keV}$

- Density: $N_{0}=\beta(s) \times\left(B_{0}^{2} / 2 \mu_{0}\right) /\left(T_{0}^{e}+T_{0}^{i}\right)$

- Major Radius : $R=2.0 \mathrm{~m}$

- Minor Radius : $a=0.5 \mathrm{~m}$

- Radial coordinate : $s=\rho / a, s_{0}=0.6$

- $L_{n 0}=0.2 m, L_{T 0}=0.08, m \rightarrow \eta_{i, e}\left(s_{0}\right)=2.5$

- $\tau(s)=\frac{T_{e}(s)}{T_{i}(s)}=1, \epsilon_{n}=\frac{L_{n 0}}{R}=0.1, \epsilon_{T}=\frac{L_{T 0}}{R}=0.04$. Shear $\hat{s}$ is positive and at $s=s_{0}, \hat{s}=1$.

\section{Equilibrium Profiles:}

- N-profile and T-profile

$$
\begin{aligned}
& N(s) / N_{0}=\exp \left(-\frac{a \delta s_{n}}{L_{n 0}} \tanh \left(\frac{s-s_{0}}{\delta s_{n}}\right)\right) \\
& T_{i, e}(s) / T_{0}=\exp \left(-\frac{a \delta s_{T}}{L_{T 0}} \tanh \left(\frac{s-s_{0}}{\delta s_{T}}\right)\right) \\
& \delta s_{n}=0.35, \delta s_{T}=0.2 \text { at } s=s_{0}
\end{aligned}
$$

- q-profile and $\hat{s}$-profile:

$q(s)=1.25+0.67 s^{2}+2.38 s^{3}-0.06 s^{4}$

such that $q\left(s=s_{0}\right)=2.0$;

The corresponding profiles for density and temperature for ions and electrons are shown in Fig. 1. The left panel shows temperature while the right panel shows the densities for the species. Note that the temperature and density profile are normalized by their respective equilibrium values i.e. $T_{0}$ and $N_{0}$. The value of $N_{0}$ can be calculated using the expression given in the Table 1 for Parameters and Profiles. The left panel of Fig. 2 shows the $\eta_{i}$
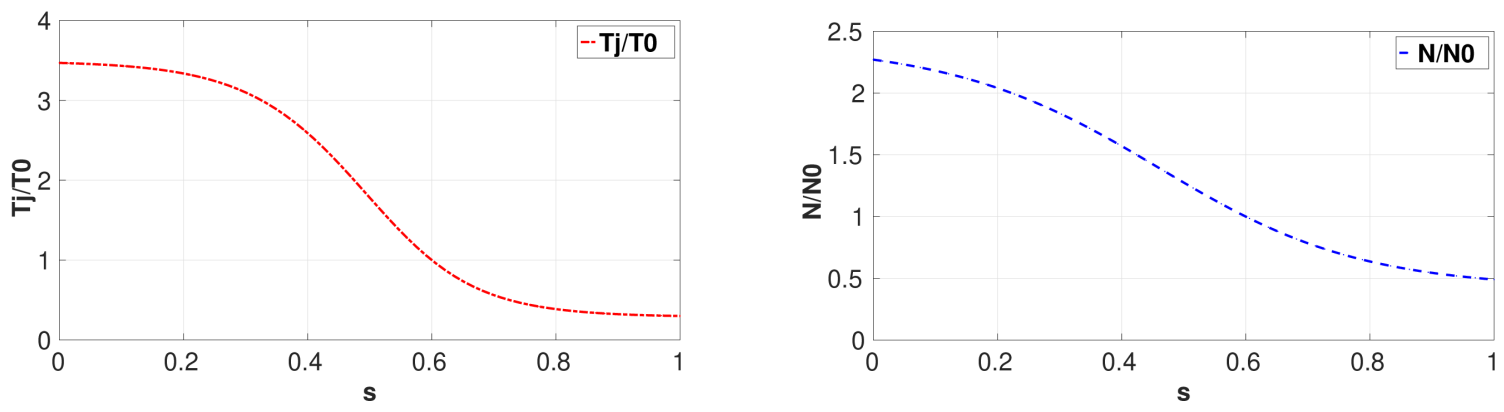

FIG. 1. Temperature profiles for electron and ion (left panel) and density profile (right panel).

profile. It is clear that it peaks at $s_{0}=0.6$. The $\eta_{i}$ profile is important, as this parameter determines the instability drive for the ITG and SWITG modes. The safety factor and shear profiles are depicted in the right panel of Fig. 2. 

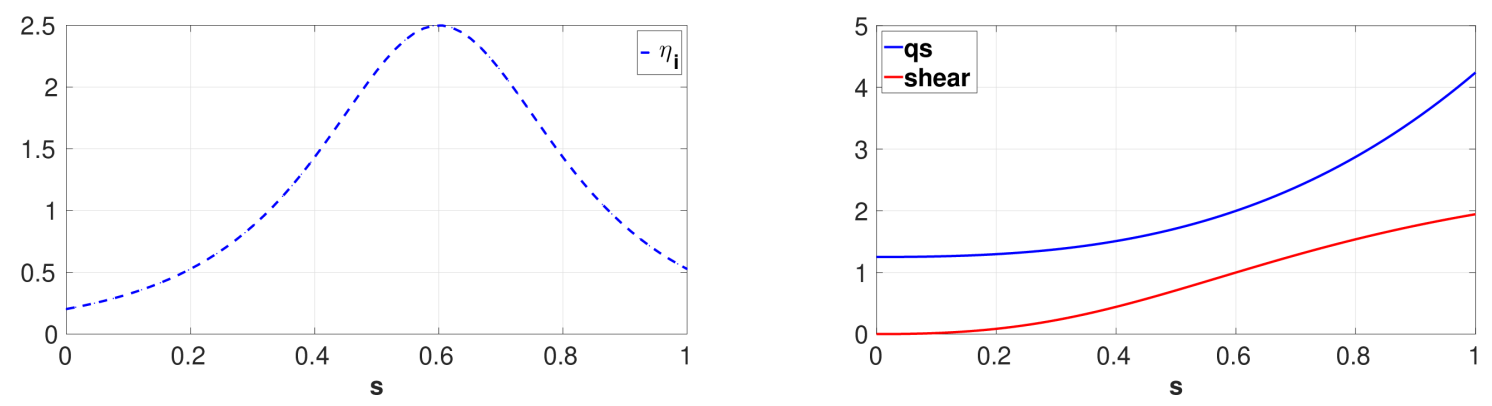

FIG. 2. $\eta_{i}$ profile (left panel) and safety factor and shear profiles (right panel).

\section{Dispersion relation}

In Fig. 3 the real frequencies and growth rates are plotted for different values of the toroidal mode number $\mathrm{n}$ and the corresponding $k_{\theta} \rho_{L i}$ values. Here the poloidal wave number $k_{\theta}=$ $\frac{m}{r}=\frac{n q\left(s_{0}\right)}{s_{0} a}$, where $m$ is the poloidal mode number. For all the simulation, poloidal mode number $(\mathrm{m})$ has been varied from $\left(n q\left(s_{0}\right)-20\right)$ to $\left(n q\left(s_{0}\right)+20\right)$. The real frequency and growth rates are normalized by $\omega_{d 0}=v_{t h j} \rho_{L i} / a^{2}$. These calculations are carried out for different values of $\beta$, namely, 0.0001 (red curve), 0.0005 (green curve) and 0.001 (blue curve). It is clear from the figure in the left panel that the real frequency increases with respect
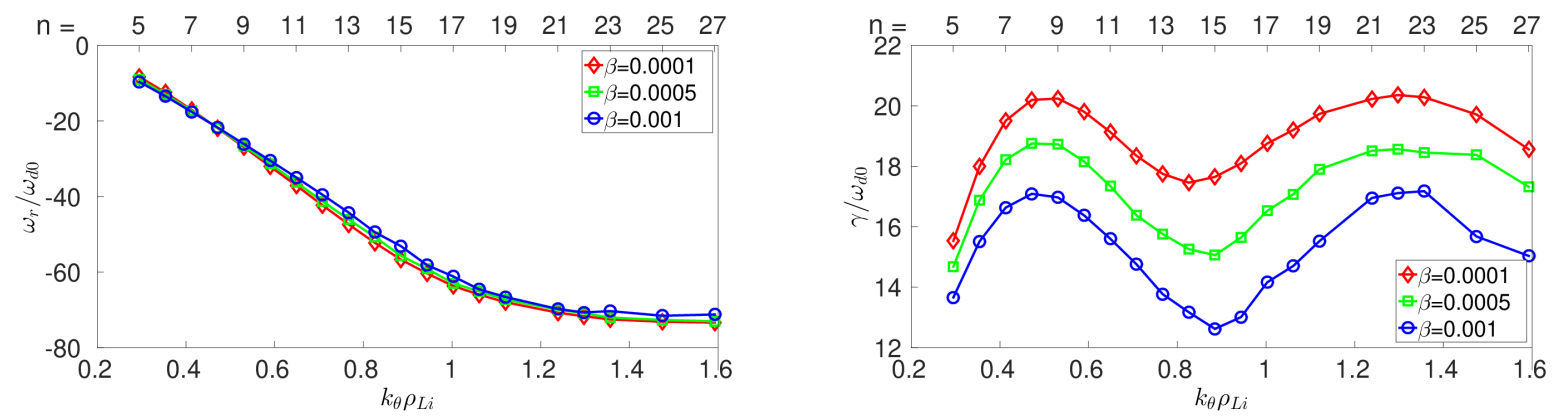

FIG. 3. Real frequency (left panel) and growth rate (right panel) with respect to $k_{\theta} \rho_{L i}$ for different $\beta$ values. The upper axis shows the corresponding toroidal mode numbers. The $\beta$ values considered are 0.0001 (red curve), $0.0005 \simeq m_{e} / m_{i}$ (green curve) and 0.001 (blue curve).

to the toroidal mode number $n$. The real frequencies increase up to toroidal mode number $n \simeq 20$. Beyond this point, the real frequencies stay virtually constant with $n$. This behavior is typical of the SWITG mode [37]. It is also evident that the real frequencies are weakly affected by the increase in $\beta$. The right panel of Fig. 3 shows the corresponding growth rates with increasing toroidal mode number $n$. The growth rate increases initially with respect to $n$ and peaks around $k_{\theta} \rho_{L i}=0.5(n \simeq 9)$ and then falls off with increasing $n$. This is the 
conventional ITG mode: with increasing toroidal mode number the resonance between mode frequency and magnetic drift enhances and therefore the growth rate increases. Beyond the peak, the resonance becomes weaker and the growth rate decreases. Also with the increase in the toroidal mode number, the finite Larmor radius effect comes in to play, because for a given ion Larmor radius with increasing toroidal mode number the wavelength decreases. However, with further increase in the toroidal mode number the real frequency turns to saturate and the growth rate again starts to increase. This is the regime of the SWITG mode. The growth rate increases up to $k_{\theta} \rho_{L i}=1.22(n \simeq 21)$ after which the growth rate again starts to decrease giving rise to the second hump which is the characteristic of the SWITG mode. The peak growth rates for both conventional ITG and SWITG mode are of similar strength. Before discussing the electromagnetic effects on the mode it is an appropriate place to revisit the theory of the SWITG mode following Ref. [37]. The perturbed distribution function for ions in the local limit $k_{\perp} \simeq k_{\theta}$ can be written as follows.

$$
f_{i}=-\frac{q_{i} f_{M i}}{T_{i}} \tilde{\phi}+\frac{q_{i} f_{M i}}{T_{i}}\left(\frac{\omega-\omega_{* i}}{\omega-\omega_{d i}-k_{\|} v_{\mid}}\right) J_{0}^{2}\left(k_{\perp} \rho_{L i}\right) \tilde{\phi}
$$

The first term represents the adiabatic response while the second term represents the nonadiabatic response of the ions. Upon integrating the perturbed distribution function with respect to velocity in the limit $\omega_{n}>\omega>\left(\omega_{d i}+k_{\|} v_{\|}\right)$, one can write the perturbed ion density in the electrostatic limit as follows [37]

$$
\tilde{n}_{i}=-\frac{q_{i} n_{o}}{T_{i}} \tilde{\phi}+\frac{q_{i}}{T_{i}} \tilde{\phi} \frac{\omega_{n i}\left(\eta_{i} / 2-1\right)}{\omega} I_{o}\left(k_{\perp}^{2} \rho_{L i}^{2}\right) \exp \left(-k_{\perp}^{2} \rho_{L i}^{2}\right)
$$

where $I_{o}$ stands for the zeroth order modified Bessel function and $\omega_{n i}=-\left(v_{t h i} / L_{n}\right)\left(k_{\theta} \rho_{L i}\right)$. Then applying the quasi-neutrality condition with adiabatic electrons one arrives at the following relation

$$
\omega=\left(\frac{\tau}{\tau+1}\right)\left(\frac{\eta_{i}}{2}-1\right) \omega_{n i} I_{o}\left(k_{\perp}^{2} \rho_{L i}^{2}\right) \exp \left(-k_{\perp}^{2} \rho_{L i}^{2}\right)
$$

The monotonic increase in the mode frequency with toroidal mode number at lower $n$ region (conventional ITG) and then saturation of the mode frequency at higher $n$ (SWITG) are easily understood from the above relation using the properties of the scaled modified Bessel function $\left.I_{o}(b) e^{-b} \rightarrow 1 / \sqrt{(} 2 \pi b\right)$ for large values of $b$. For small $k_{\perp}^{2} \rho_{L i}^{2}$, the mode frequency 
$\omega$ behaves as $k_{\perp} \rho_{L i}$ and for larger $k_{\perp}^{2} \rho_{L i}^{2}$ it remains virtually constant. The second hump in the growth rate appears as a result of a second resonance between the toroidal magnetic drift term $\omega_{d i}$ of the ions with the mode frequency which is constant at higher toroidal mode number. It is also clear from the Fig. 3 that the SWITG also suffers from finite Larmor radius stabilization. This can be understood from the expression of the nonadiabatic part in Eq.6. At very high $n$ or equivalently, high $k_{\perp} \rho_{L i}$, the ion magnetic drift frequency $\omega_{d i}$ leads the mode frequency $\omega$. The nonadiabatic part of the perturbed ion density, in the limit $\omega_{d i}>>\omega$ and large $k_{\perp}^{2} \rho_{L i}^{2}$ will then decrease as $\frac{R}{L_{n}} I_{o}\left(k_{\perp}^{2} \rho_{L i}^{2}\right) \exp \left(-k_{\perp}^{2} \rho_{L i}^{2}\right)$. For greater detail of these calculations, readers are referred to Ref [37]. It is evident from Fig. 3 that, although the real frequencies are not very much affected by the increase in the value of $\beta$, the growth rates of both conventional ITG and SWITG mode suffer a substantial reduction in magnitude. The reduction in the growth rates of the conventional ITG mode is well known. There have been many linear and nonlinear studies reporting the stabilization of the ITG mode by the electromagnetic perturbations. However, the role of electromagnetic perturbations on the SWITG mode is perhaps hitherto not investigated in detail using global gyrokinetic model. Since the advanced operating regimes where SWITG mode can be unstable also have higher $\beta$ values, it is important to explore the electromagnetic effect on the mode. Thus this present study shows that the SWITG mode suffers stabilization in the presence of the electromagnetic perturbation and one has to take in to account this effect as well when studying the ion heat flux induced by SWITG in real experiments. In the presence of the electromagnetic perturbation, the mode couples with the Alfven perturbations. The field lines are thus bent in the presence of $\beta$ [49, [55]. This field line bending by the electromagnetic perturbation is therefore responsible for the stabilization of the SWITG mode. The stabilization by field line bending may be understood looking at the energy equation for stability in the ideal MHD theory. If $\delta W$ is the change in the potential energy under a small perturbation, the condition $\delta W<0$ leads to an unstable situation. If we retain only field line bending and pressure driven terms for simplicity, then $\delta W$ can be written as $\delta W=\int d v\left[\frac{|\tilde{B}|^{2}}{2 \mu_{0}}-\left(\xi_{\perp} \cdot \nabla p\right)\left(\kappa \cdot \xi_{\perp}^{*}\right)\right]$, where $\tilde{B}, p, \xi_{\perp}$ and $\kappa$ are perturbed magnetic field, pressure, plasma displacement and curvature, respectively. The first term which comes from field line bending is always positive and therefore provides stabilization. The mode averaged wavenumbers versus toroidal mode number $n$ is shown in Fig. 4. It is clear from the figure that with the increase in $n$, the value of $\left\langle\kappa \rho_{L i}\right\rangle$ increases initially up to $n \simeq 15$, 
but with further increase in the value of $n$, SWITG branch starts (see Fig. 3) and $\left\langle\kappa \rho_{L i}\right\rangle$ tends to saturates. Similar to the ITG branch, for SWITG branch as well, the contribution of $\left\langle\kappa \rho_{L i}\right\rangle$ to $\left\langle k_{\perp} \rho_{L i}\right\rangle$ is significant. It is also important to note that with the increase in $\beta$ values, $\left\langle k_{\theta} \rho_{L i}\right\rangle$ value does not change much, but $\left\langle\kappa \rho_{L i}\right\rangle$ increases. Hence, for higher $\beta$ values, the relative contribution of $\left\langle\kappa \rho_{L i}\right\rangle$ to $\left\langle k_{\perp} \rho_{L i}\right\rangle$ also increases. For example, for $n=21$, $\left\langle k_{\theta} \rho_{L i}\right\rangle=1.571,1.560,1.556$ and $\left\langle\kappa \rho_{L i}\right\rangle /\left\langle k_{\perp} \rho_{L i}\right\rangle=0.446,0.484,0.606$ for respective values of $\beta=0.0001,0.005,0.001$. This suggests the radial length scale becomes shorter and shorter for higher $\beta$ values and also comparable to the poloidal length scale. This strong radial behavior shows the kinetic as well as global nature of the SWITG mode.

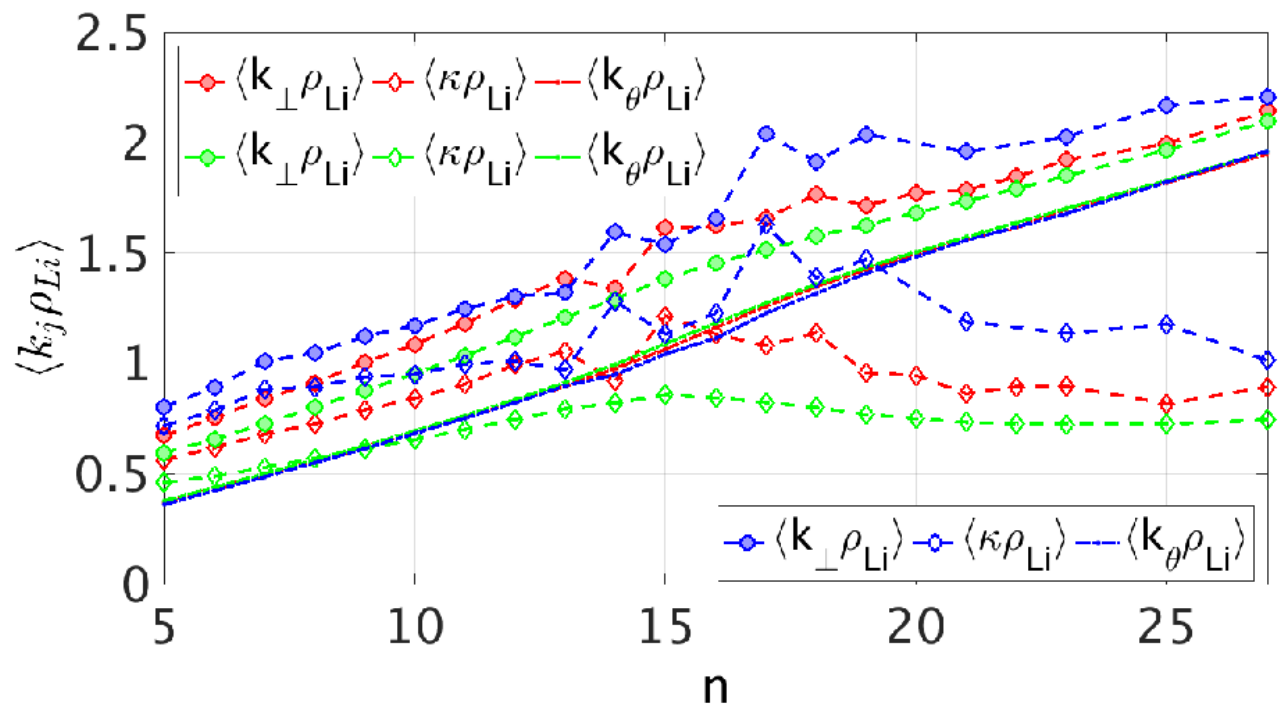

FIG. 4. Mode-averaged wavenumbers versus toroidal mode number $n$ for $\beta=0.0001$ (green color), $\beta=0.0005$ (red color), $\beta=0.001$ (blue color).

\section{A. Mode structures for ITG and SWITG}

Fig. 5 shows the mode structures for electrostatic and electromagnetic potentials $\phi$ and $A_{\|}$ for $\beta=0.0001$. The toroidal mode number corresponding to the mode structure is $n=9$. It is clear from the figure that for both $\phi$ and $A_{\|}$the mode structures exhibit ballooning character. Similarly, Fig. 6 depicts the mode structure for $\phi$ and $A_{\|}$for $\beta=0.001$ for toroidal mode $n=9$. Similar to the modes in Fig. 5, the modes exhibit ballooning structure which is a characteristic of ITG mode. Figs. 7 and 8 show the mode structures of $\phi$ and $A_{\|}$ 

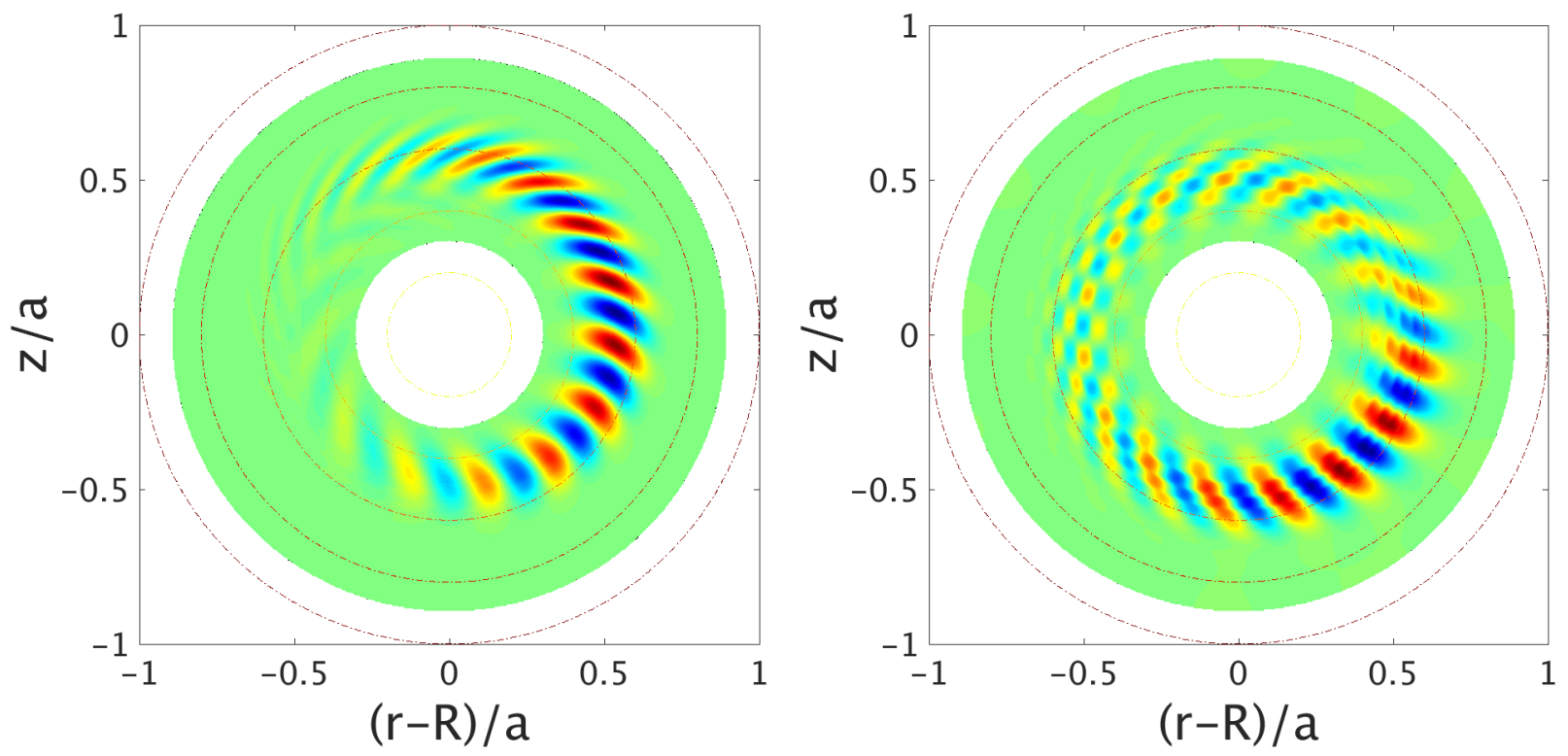

FIG. 5. Mode structure of $\phi$ (left panel) and $A_{\|}$(right panel) for $n=9$ corresponding to the ITG mode $\beta=0.0001$.
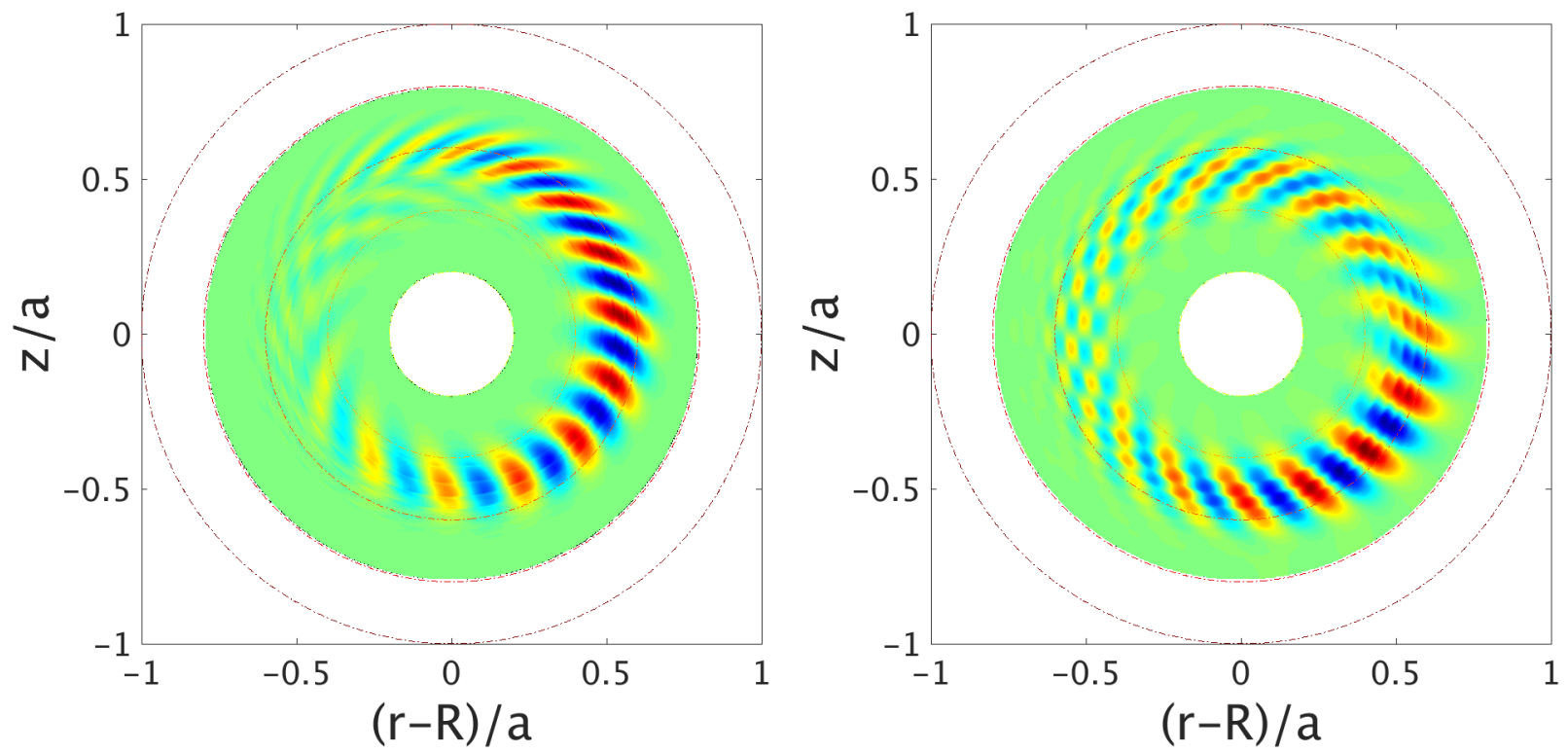

FIG. 6. Mode structure of $\phi$ (left panel) and $A_{\|}$(right panel) for $n=9$ corresponding to the ITG mode $\beta=0.001$.

for SWITG mode for $\beta=0.0001$ and $\beta=0.001$, respectively. The toroidal mode number for these figures is $n=21$. The figures clearly show that the SWITG mode is ballooning in nature and more localized both radially and poloidally as compared to the normal ITG mode. This observation suggests that the mode might be susceptible to stabilization due to toroidicity [39]. 

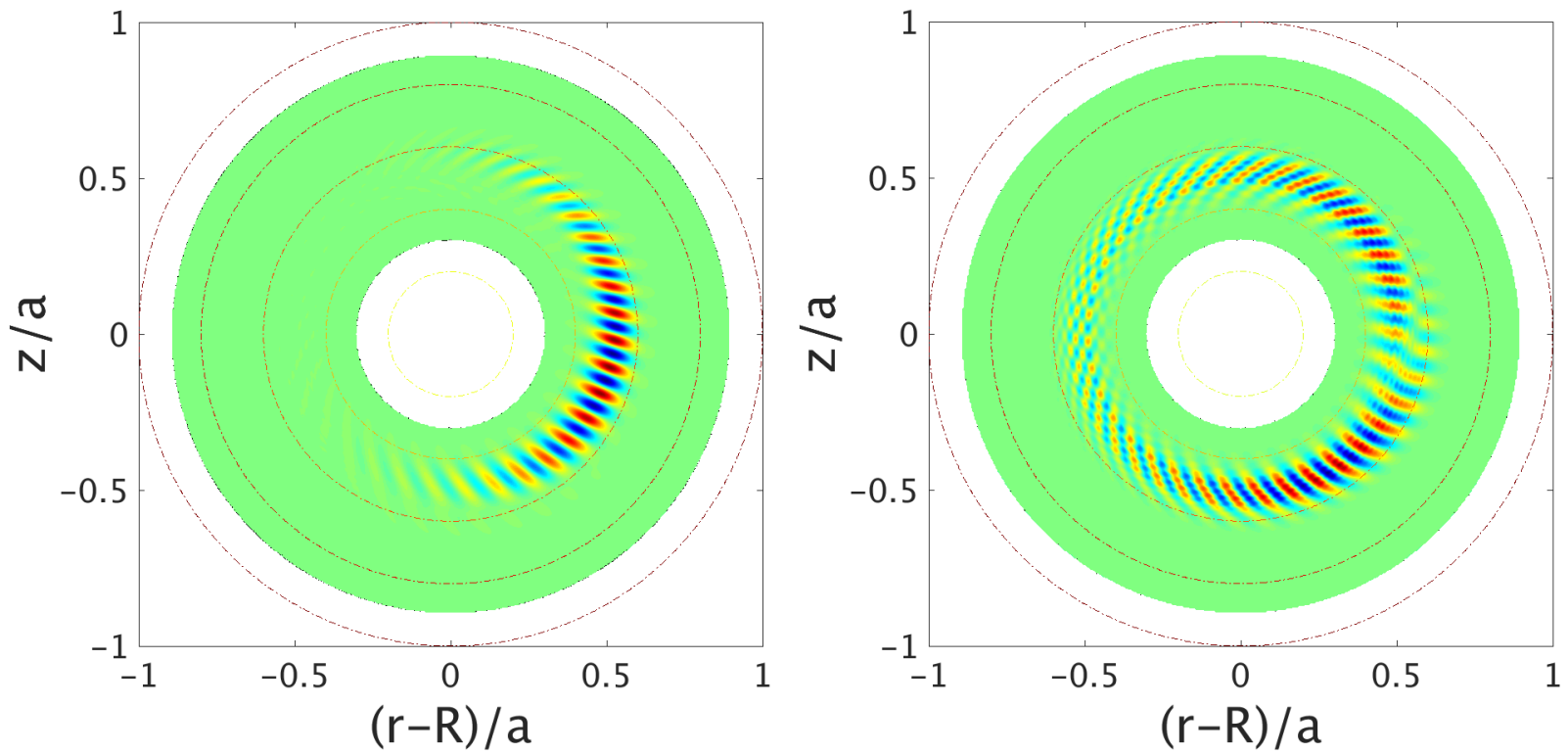

FIG. 7. Mode structure of $\phi$ (left panel) and $A_{\|}$(right panel) for $n=21$ corresponding to the SWITG mode for $\beta=0.0001$.
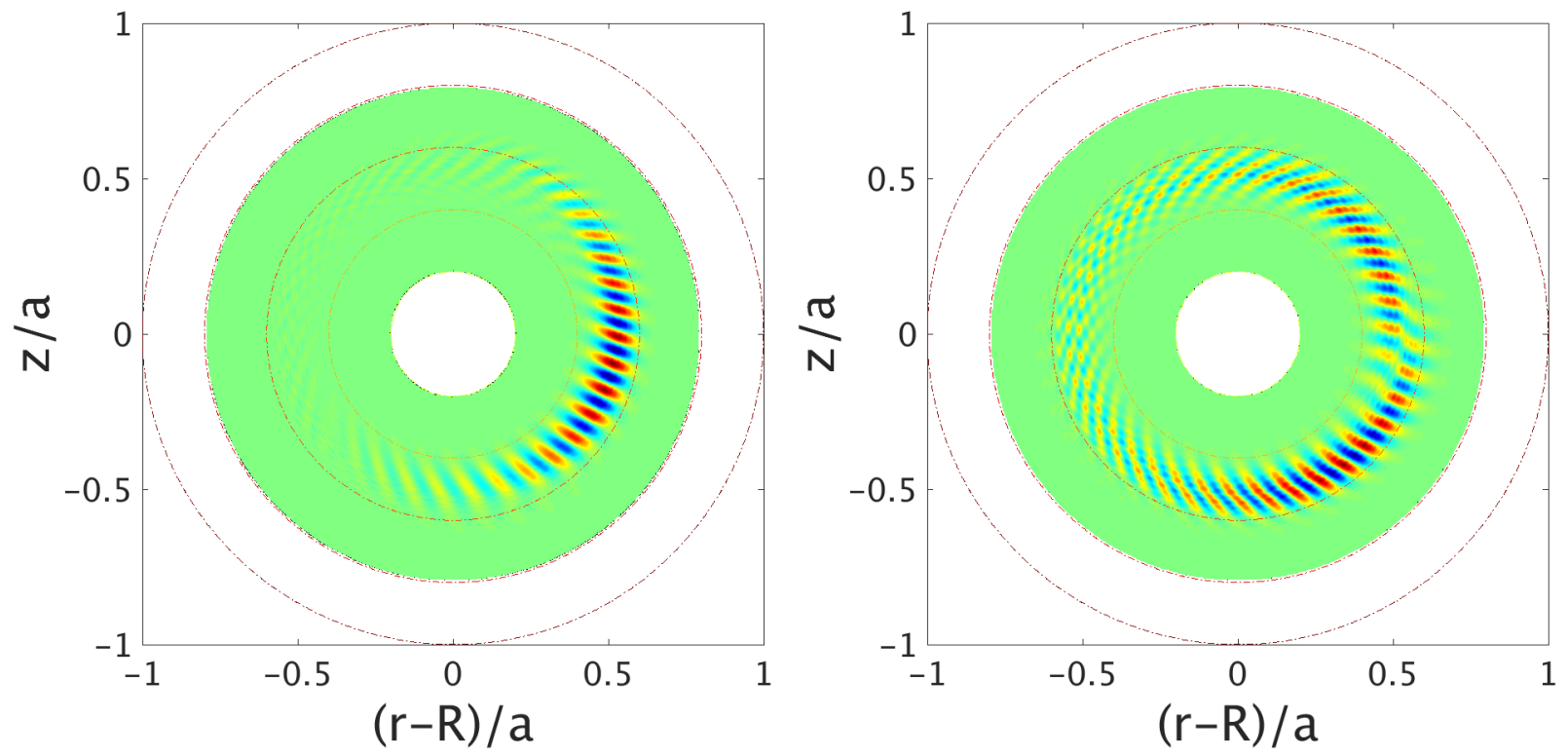

FIG. 8. Mode structure of $\phi$ (left panel) and $A_{\|}$(right panel) for $n=21$ corresponding to the SWITG mode for $\beta=0.001$.

\section{B. $\beta$ scan}

In this section, we investigate the effect of $\beta$ on the real frequency and growth rates of SWITG mode and compare them with the conventional ITG mode. The real frequencies and growth rates are calculated for increasing values of $\beta$ and are shown in Fig. 9. The real 
frequencies versus $\beta$ are shown in the left panel and the growth rates versus $\beta$ are shown in the right panel. It is clear from the figure that with increasing $\beta$ the real frequency increases weakly with $\beta$. In contrast to this, the growth rate decreases with increasing $\beta$. However, at a higher $\beta$ growth rate of SWITG mode decreases slowly with respect to $\beta$ compared to that of ITG mode. Thus we can conclude that the SWITG mode suffers $\beta$ stabilization
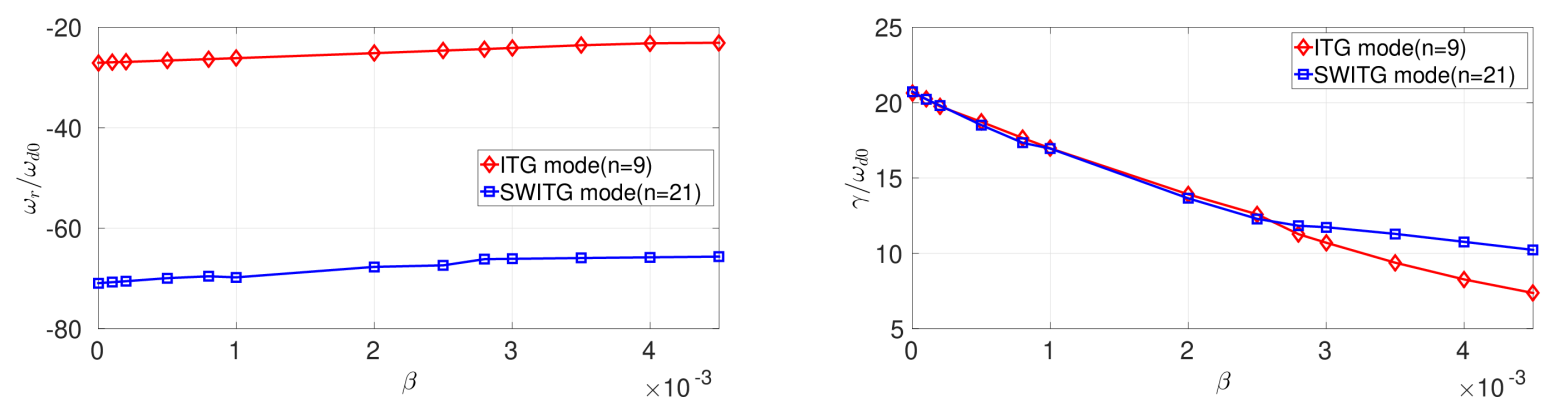

FIG. 9. Real frequency (left panel) and growth rates (right panel) for the ITG and SWITG mode with respect to $\beta$.

like its long wavelength counterpart. The relative strength of the electromagnetic to the electrostatic character is shown in Fig. 10. This is expressed as the ratio $\left\langle A_{\|}\right\rangle^{2} /\langle\phi\rangle^{2}$. It is clear from the figure that the ratio increases almost linearly with increasing $\beta$. It is also observed that the value of the $\left\langle A_{\|}\right\rangle^{2} /\langle\phi\rangle^{2}$ ratio for SWITG is lower compared to that of the conventional ITG mode. Here, the value of $m_{i} / m_{e}$ is 1836 .

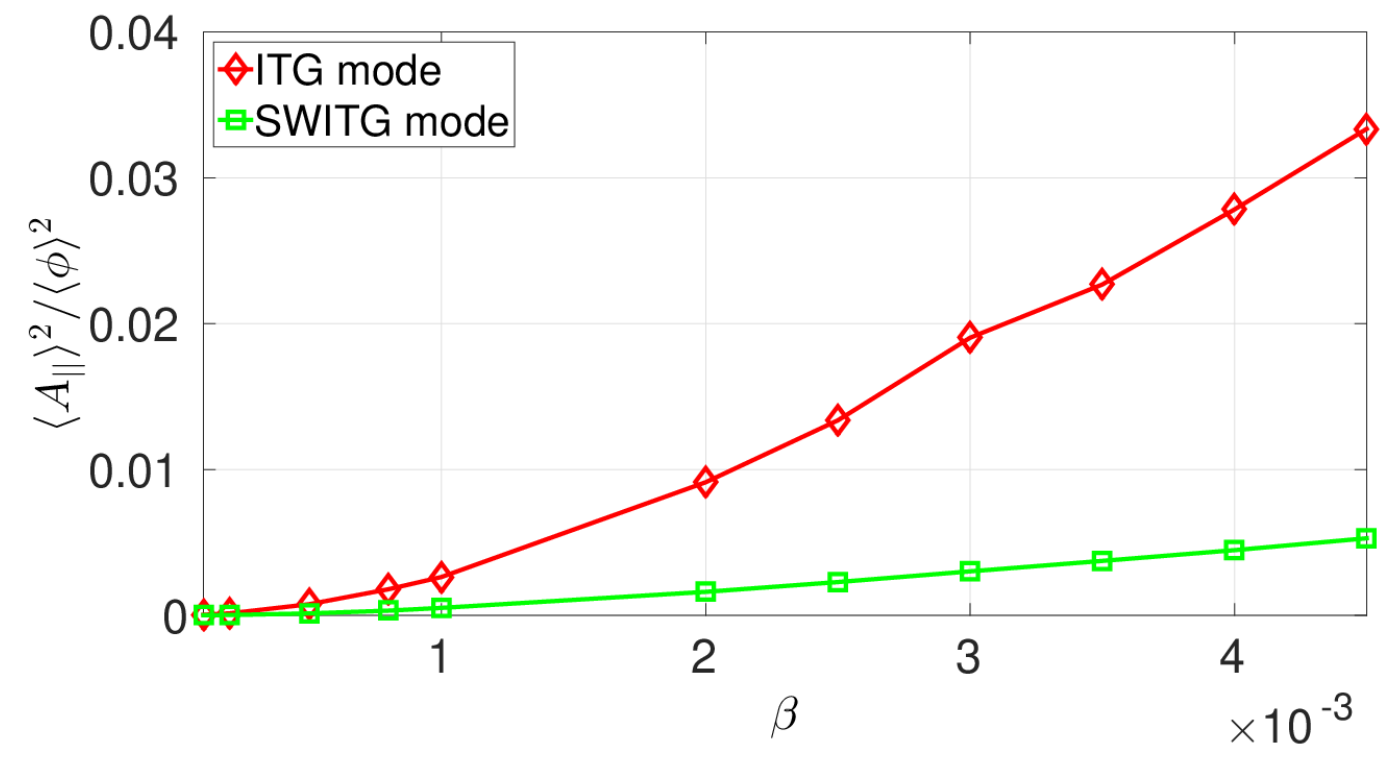

FIG. 10. Ratio of $\left\langle A_{\|}\right\rangle^{2} /\langle\phi\rangle^{2}$ with respect to $\beta$ for the ITG and SWITG mode. 


\section{C. $\eta_{i}$ scan}

For temperature gradient modes, such as the SWITG mode, $\eta_{i}$, which is the ratio of density and temperature gradient scale lengths, is a very important parameter. When $\eta_{i}$ is above a certain value the SWITG mode becomes unstable. Beyond the threshold value, the growth rate increases monotonically with increasing $\eta_{i}$. To explore this physics in the presence of electromagnetic perturbation, we calculate the mode frequency and growth rate for increasing values of $\eta_{i}$ for both the ITG and the SWITG modes for different values of $\beta$. The results for the ITG mode are shown in Fig. 11 and the corresponding results for the SWITG mode are shown in Fig. 12 .
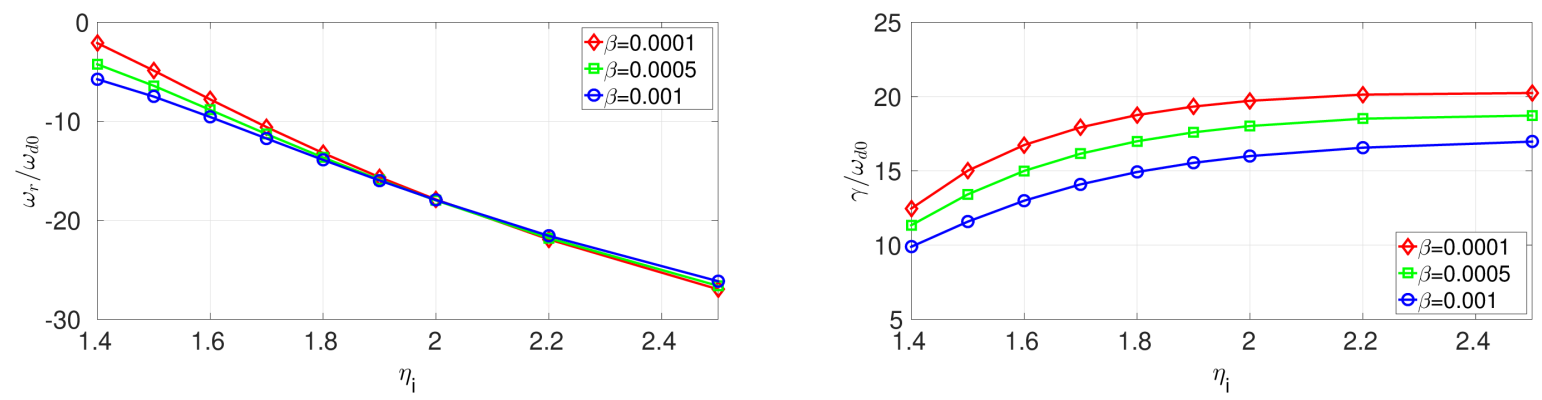

FIG. 11. Real frequency (left panel) and growth rates (right panel) with respect to $\eta_{i}$ for the ITG mode.
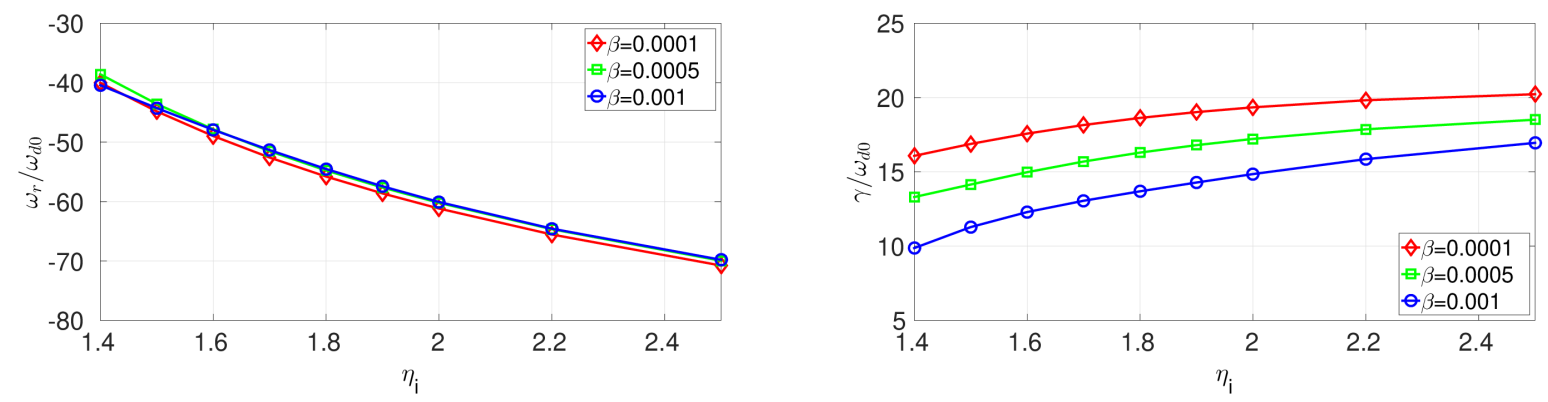

FIG. 12. Real frequency (left panel) and growth rates (right panel) with respect to $\eta_{i}$ for the SWITG mode.

The growth rates increase with $\eta_{i}$ in both the cases. An explanation for the $\eta_{i}$ dependence of the ITG and SWITG mode can be given following Ref.[55]. The free energy for ITG mode and SWITG mode comes from the temperature gradient. In the presence of density gradient, simultaneous convection in temperature and density gradients takes place resulting in a competition between convection and expansion in the energy equation. During the outward 
convection, plasma moves from a higher density region to a lower density region where expansion takes place. At the same time, plasma moves from a higher temperature region to a lower temperature region increasing the temperature. The expansion leads to the cooling of the plasma that competes with the increase in the temperature. For a displacement $\xi$, the change in the temperature can be written as [55], $\delta T=-\xi \cdot \nabla T+\alpha \xi \cdot \nabla n$, where $\alpha$ is

a coefficient giving the cooling due to expansion. This relation clearly shows that for $\delta T$ to be positive, $\eta$ has to exceed a certain threshold value. The net free energy available depends upon the relative strength of the density gradient and temperature gradient [55]. This is manifested in the dependence on $\eta_{i}$. Thus with increasing $\eta_{i}$ the free energy available to render the mode unstable also increases. This leads to an increase in the growth rate. This explains the increase in the growth rates with increasing $\eta_{i}$ as observed in Figs. 11 and 12 , It is also evident from the Figs. 11 and 12 that the real frequency increases with $\eta_{i}$. The real frequency is proportional to the diamagnetic frequency which is proportional to the gradient. That is why the real frequency increases with increasing $\eta_{i}$.

\section{Mixing length calculation of flux}

It would be interesting to see how the electromagnetic perturbation affects the overall flux. Since this is a linear simulation, one can use a simple mixing length estimation of the transport coefficient. For the purpose, we calculate the ratio $D_{M L}=\gamma /\left\langle k_{\perp}^{2}\right\rangle$. Here, $\gamma$ is the growth rate for a given $n$ and the mode square average of the perpendicular wave-vector $\left\langle k_{\perp}^{2}\right\rangle$ $=\left\langle\kappa^{2}\right\rangle+\left\langle k_{\theta}^{2}\right\rangle$. Calculation of the mode square average of radial and poloidal wave-vectors are being done using the following expressions.

$$
\begin{gathered}
\left\langle\kappa^{2}\right\rangle=\frac{\Sigma_{(k, m)}|\kappa \phi|^{2}+\left|\kappa A_{\|}\right|^{2}}{\Sigma_{(k, m)}|\phi|^{2}+\left|A_{\|}\right|^{2}} \\
\left\langle k_{\theta}^{2}\right\rangle=\frac{\int d r \Sigma_{m}\left|\frac{m}{r} \phi_{(k, m)}\right|^{2}+\left|\frac{m}{r} A_{\|(k, m)}\right|^{2}}{\int d r \Sigma_{m}\left|\phi_{(k, m)}\right|^{2}+\left|A_{\|(k, m)}\right|^{2}}
\end{gathered}
$$

In Fig. 13 we calculate the quantity $D_{M L}$ for each value of toroidal mode number and plot the same with respect to the toroidal mode number. We consider three cases of $\beta$ values, $\beta=0.0001,0.0005$ and 0.001 corresponding to the results shown in Fig. 3. It is observed in Fig. 13 that the mixing length estimation of heat flux peaks at the longest wavelength 
despite the fact that the SWITG mode exhibits the strongest growth rates around $n=21$. This implies that most of the contribution to the total flux comes from modes close to the conventional ITG. This is qualitatively in conformity with the results observed in Ref. [40]. It is to be noted that with increasing value of $\beta$ the magnitude of $\gamma /\left\langle k_{\perp}^{2}\right\rangle$ decreases implying a reduction in the heat flux. This is consistent with the stabilization effect observed in Fig. 3 and Fig. 9 with respect to increasing $\beta$. Thus we conclude that increasing $\beta$ does not only reduce growth rates of the mode but might also reduce the overall heat flux.

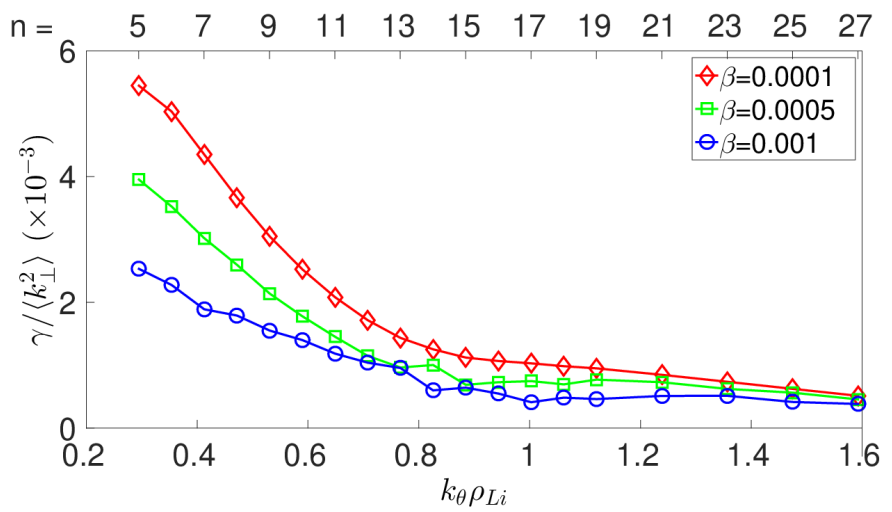

FIG. 13. Mixing length estimate for transport coefficient $D_{M L}=\gamma /\left\langle k_{\perp}^{2}\right\rangle$ with respect to $k_{\theta} \rho_{L i}$. The upper $\mathrm{x}$-axis shows the corresponding toroidal mode number $n$. Three values of $\beta=0.0001,0.0005$ and 0.001 are considered.

\section{SUMMARY}

In the present work, we have carried out a systematic study of the electromagnetic effect on the SWITG mode along with the conventional ITG mode using a global linear gyrokinetic model. Although the electromagnetic effect on the ITG mode is hitherto well known, this is the first study that investigates the electromagnetic effects on the SWITG mode using global gyrokinetic simulations. We have calculated the real frequency and growth rate for the chosen equilibrium for three different values of $\beta$. We also have shown the mode structures for both the ITG and the SWITG mode in the presence of finite $\beta$. We have found out the growth rates and real frequencies with respect to $\beta$ and with increasing $\eta_{i}$ for different values of $\beta$. Finally, we have presented the mixing length estimation of the transport. The main findings are as follows. 
1. The SWITG mode is stabilized by the electromagnetic effect. The real frequency of the SWITG mode is weakly affected by the electromagnetic effect.

2. The SWITG mode-structure is more localized both radially and poloidally compared to the ITG mode.

3. With increasing $\beta$ the real frequency of the SWITG mode decreases slightly while the growth rate decreases substantially.

4. For the range of $\eta_{i}$ values studied, the growth rate of the SWITG mode instability decreases with an increase in $\beta$ value.

5. The ratio of electromagnetic to electrostatic potentials increases with increasing $\beta$.

6. The contribution of $\left\langle\kappa \rho_{L i}\right\rangle$ value is significant, compared to that of $\left\langle k_{\theta} \rho_{L i}\right\rangle$, for the calculation of $\left\langle k_{\perp} \rho_{L i}\right\rangle$. Also, with the increase in $\beta$ value, this contribution increases.

7. The mixing length estimate for transport reveals that although the linear growth rates of the SWITG mode are comparable to those of the long wavelength branch the heat flux is maximum at the long wavelength region. This implies that most of the contribution to the ion heat flux still comes from the conventional ITG mode that occurs at $k_{\theta} \rho_{i} \leqslant 1.0$.

8. The mixing length estimate for transport shows that with increasing $\beta$ the heat flux decreases. This is in conformity with the linear stabilization of the mode with increasing $\beta$.

As is well known, in the large aspect ratio limit, the trapped electron fraction goes as $\sqrt{2 \epsilon}$ where $\epsilon$ is the inverse aspect ratio $a / R_{0}$. Thus for typical aspect ratio considered here, effect of trapped electrons on finite $\beta$ SWITG may become important. It would therefore make sense to investigate this effect by considering the high $\beta$ version of the code EMGLOGYSTO, wherein dynamics due to $\left(\phi, A_{\|}, A_{\perp}\right)$ perturbations may be addressed. This will be attempted in near future. 


\section{ACKNOWLEDGEMENT}

All the simulations reported here are performed using the Udbhav cluster at the Institute for Plasma Research(IPR). This paper is dedicated to late Professor Jan Vaclavik who taught one of the authors $(\mathrm{RG})$ gyrokinetic theory.

\section{DATA AVAILABILITY}

The data that support the findings of this study are available from the corresponding author upon reasonable request.

\section{Appendix A}

The set of equations discussed in Section II are closed by invoking the quasi-neutrality condition and the component of Ampère's law parallel to B:

$$
\sum_{j} \tilde{n}_{j}(\mathbf{r} ; \omega) \simeq 0 ; \quad \frac{1}{\mu_{0}} \nabla_{\perp}^{2} \tilde{A}_{\|}=-\sum_{j} \tilde{j}_{\| j}
$$

Equation (A1) defines a generalized eigenvalue problem with eigenvalue $\omega$. This eigenvalue problem is conveniently solved in Fourier space. By Fourier decomposing the potential in Eq.A1 and then taking Fourier transform, we obtain a convolution matrix in Fourier space. If we assume a hydrogen-like plasma with ions, electrons, we have:

$$
\sum_{\mathbf{k}^{\prime}}\left(\begin{array}{cc}
\sum_{j=i, e} \mathcal{M}_{\tilde{\varphi} \tilde{\varphi}, \mathbf{k}, \mathbf{k}^{\prime}}^{j} & \sum_{j=i, e} \mathcal{M}_{\tilde{\varphi} \tilde{\varphi} \tilde{A}_{\|}, \mathbf{k}, \mathbf{k}^{\prime}}^{j} \\
\sum_{j=i, e} \mathcal{M}_{\tilde{A}_{\|} \tilde{\varphi}, \mathbf{k}, \mathbf{k}^{\prime}}^{j} & \sum_{j=i, e} \mathcal{M}_{\tilde{A}_{\|} \tilde{A}_{\|}, \mathbf{k}, \mathbf{k}^{\prime}}
\end{array}\right)\left(\begin{array}{c}
\tilde{\varphi}_{\mathbf{k}^{\prime}} \\
\tilde{A}_{\|, \mathbf{k}^{\prime}}
\end{array}\right)=0
$$

where $\mathbf{k}=(\kappa, m)$ and $\mathbf{k}^{\prime}=\left(\kappa^{\prime}, m^{\prime}\right)$. Note that we have 2 species: passing ions $(i)$ and passing electrons $(e)$. In the following, we discuss in detail the formulation for passing species. The Laplacian for the parallel component of Ampère's law is also included in the appropriate matrix elements. The submatrices $\mathcal{M}$ are symmetric about the diagonal. With the following definitions, $\Delta \rho=\rho_{u}-\rho_{l}$ (upper and lower radial limits), $\Delta_{\kappa}=\kappa-\kappa^{\prime}$ and $\Delta_{m}=m-m^{\prime}$ 
matrix elements are :

$$
\begin{aligned}
& \mathcal{M}_{\tilde{\varphi} \tilde{\varphi}, \mathbf{k}, \mathbf{k}^{\prime}}^{i}=\frac{1}{\Delta r} \int_{r_{l}}^{r_{u}} d r \exp \left(-i \Delta_{\kappa} r\right) \times\left[\alpha_{p} \delta_{m m^{\prime}}+\exp \left(i \Delta_{m} \bar{\theta}\right) \sum_{p} \hat{I}_{p, i}^{0}\right], \\
& \mathcal{M}_{\tilde{\varphi} \tilde{A} \|, \mathbf{k}, \mathbf{k}^{\prime}}^{i}=-\frac{1}{\Delta r} \int_{r_{l}}^{r_{u}} d r \exp \left(-i \Delta_{\kappa} r\right) \times\left[\exp \left(i \Delta_{m} \bar{\theta}\right) \sum_{p} \hat{I}_{p, i}^{1}\right], \\
& \mathcal{M}_{\tilde{\varphi} \tilde{\varphi}, \mathbf{k}, \mathbf{k}^{\prime}}^{e}=\frac{1}{\Delta r} \int_{r_{l}}^{r_{u}} d r \exp \left(-i \Delta_{\kappa} r\right) \times\left[\frac{\alpha_{p} \delta_{m m^{\prime}}}{\tau(r)}+\frac{\exp \left(i \Delta_{m} \bar{\theta}\right)}{\tau(r)} \sum_{p} \hat{I}_{p, e}^{0}\right], \\
& \mathcal{M}_{\tilde{\varphi} \tilde{A}_{\|,}, \mathbf{k}, \mathbf{k}^{\prime}}^{e}=-\frac{1}{\Delta r} \int_{r_{l}}^{r_{u}} d r \exp \left(-i \Delta_{\kappa} r\right) \times\left[\frac{\exp \left(i \Delta_{m} \bar{\theta}\right)}{\tau(r)} \sum_{p} \hat{I}_{p, e}^{1}\right], \\
& \mathcal{M}_{\tilde{A}_{\|} \tilde{A}_{\|}, \mathbf{k}, \mathbf{k}^{\prime}}^{i}=\frac{1}{\Delta r} \int_{r_{l}}^{r_{u}} d r \exp \left(-i \Delta_{\kappa} r\right) \times\left[\exp \left(i \Delta_{m} \bar{\theta}\right) \sum_{p} \hat{I}_{p, i}^{2}\right] \\
&-\frac{1}{\Delta r} \int_{r_{l}}^{r_{u}} d r \exp \left(-i \Delta_{\kappa} r\right) \times\left(\kappa^{\prime 2}+\frac{m^{\prime 2}}{r^{2}}\right)\left(\frac{T_{i}(r)}{q_{i}^{2} N \mu_{0}}\right), \\
& \mathcal{M}_{\tilde{A}_{\|} \tilde{A}_{\|}, \mathbf{k}, \mathbf{k}^{\prime}}^{e}=\frac{1}{\Delta r} \int_{r_{l}}^{r_{u}} d r \frac{\exp \left(-i \Delta_{\kappa} r\right)}{\tau(r)} \times\left[\exp \left(i \Delta_{m} \bar{\theta}\right) \sum_{p} \hat{I}_{p, e}^{2}\right]
\end{aligned}
$$

where

$$
\begin{gathered}
\hat{I}_{p, j}^{l}=\frac{1}{\sqrt{2 \pi} v_{t h, j}^{3}(r)} \int_{-v_{\text {max }, j}(r)}^{v_{\max , j}(r)} v_{\|}^{l} d v_{\|} \exp \left(-\frac{v_{\|}^{2}}{v_{t h, j}^{2}(r)}\right)\left\{\frac{N_{1}^{j} I_{0, j}^{\sigma}-N_{2}^{j} I_{1, j}^{\sigma}}{D_{1}^{\sigma, j}}\right\}_{p^{\prime}=p-\left(m-m^{\prime}\right)}, \\
I_{n, j}^{\sigma}=\int_{0}^{v_{\perp m a x, j}(r)} v_{\perp}^{2 n+1} d v_{\perp} \exp \left(-\frac{v_{\perp}^{2}}{2 v_{t h, j}^{2}(r)}\right) J_{0}^{2}\left(x_{L j}\right) J_{p}\left(x_{t j}^{\prime}\right) J_{p^{\prime}}\left(x_{t j}^{\prime \sigma}\right),
\end{gathered}
$$

We have introduced the following definitions: $\epsilon$ is the inverse aspect ratio, $v_{\max , j}(r)$ is the upper cutoff speed (considered in the numerical implementation) of the species $j, v_{\perp \max , j(\rho)}=$ $\min \left(v_{\|} / \sqrt{\epsilon}, v_{\max , j}\right)$ which is "trapped particle exclusion" from $\omega$ independent perpendicular velocity integral $I_{n, j}^{\sigma} ; \alpha_{p}=1-\sqrt{\epsilon /(1+\epsilon)}$ is the fraction of passing particles; $\hat{I}_{p, j}^{l}$, is $\omega-$ dependent parallel integrals; $\left.x_{t j}^{\sigma}=k_{\perp} \xi_{\sigma}, N_{1}^{j}=\omega-w_{n, j}\left[1+\left(\eta_{j} / 2\right)\left(v_{\|}^{2} / v_{t h, j}^{2}\right)-3\right)\right] ; N_{2}^{j}=$ $w_{n, j} \eta_{j} /\left(2 v_{t h, j}^{2}\right)$ and $D_{1}^{\sigma, j}=<w_{t, j}(\rho)>\left(n q_{s}-m^{\prime}(1-p)\left(\sigma v_{\|} / v_{t h, j}\right)-\omega\right.$ where $<w_{t, j}(\rho)>=$ 
$v_{t h, j}(\rho) /\left(r q_{s}\right)$ is the average transit frequency of the species $j$.

[1] B. Coppi, M. Rosenbluth, and R. Sagdeev, Phys. Fluids 10, 582 (1967).

[2] F. Romanelli, Phys. Fluids B: Plasma Physics 1, 1018 (1989).

[3] W. Horton, Rev. Mod. Phys. 71, 735 (1999).

[4] J. Chowdhury, R. Ganesh, P. Angelino, J. Vaclavik, L. Villard, and S. Brunner, in Journal of Physics: Conference Series, Vol. 208 (IOP Publishing, 2010) p. 012058.

[5] J. Chowdhury, W. Wang, S. Ethier, J. Manickam, and R. Ganesh, Phys. Plasmas 19, 042503 (2012).

[6] B. Kadomtsev and O. Pogutse, Nucl. Fusion 11, 67 (1971).

[7] M. Rosenbluth and M. Sloan, Phys. Fluids 14, 1725 (1971).

[8] B. Coppi and G. Rewoldt, Phys. Rev. Lett. 33, 1329 (1974).

[9] P. C. Liewer, Nucl. Fusion 25, 543 (1985).

[10] J. Chowdhury, R. Ganesh, S. Brunner, J. Vaclavik, L. Villard, and P. Angelino, Phys. Plasmas 16, 052507 (2009).

[11] J. Chowdhury, W. Wang, S. Ethier, J. Manickam, and R. Ganesh, Phys. Plasmas 18, 112510 (2011).

[12] K. Tsang, P. Catto, J. Whitson, and J. Smith, Phys. Rev. Lett. 40, 327 (1978).

[13] J. Chowdhury, R. Ganesh, S. Brunner, J. Vaclavik, and L. Villard, Phys. Plasmas 17, 102105 (2010).

[14] M. Landreman, T. M. Antonsen Jr, and W. Dorland, Phys. Rev. Lett. 114, 095003 (2015).

[15] P. Guzdar, C. Liu, J. Dong, and Y. Lee, Phys. Rev. Lett. 57, 2818 (1986).

[16] Y. Lee, J. Dong, P. Guzdar, and C. Liu, Phys. fluids 30, 1331 (1987).

[17] W. Horton, B. Hong, and W. Tang, Phys. fluids 31, 2971 (1988).

[18] W. Dorland, F. Jenko, M. Kotschenreuther, and B. Rogers, Phys. Rev. Lett. 85, 5579 (2000).

[19] J. Drake and Y. Lee, Phys. Fluids 20, 1341 (1977).

[20] N. Gladd, J. Drake, C. Chang, and C. Liu, Phys. Fluids 23, 1182 (1980).

[21] H. Doerk, F. Jenko, T. Görler, D. Told, M. Pueschel, and D. Hatch, Phys. Plasmas 19, 055907 (2012). 
[22] W. Guttenfelder, J. Candy, S. Kaye, W. Nevins, E. Wang, J. Zhang, R. Bell, N. Crocker, G. Hammett, B. LeBlanc, et al., Phys. Plasmas 19, 056119 (2012).

[23] Y. Chen, J. Chowdhury, S. Parker, and W. Wan, Phys. Plasmas 22, 042111 (2015).

[24] A. K. Swamy, R. Ganesh, J. Chowdhury, S. Brunner, J. Vaclavik, and L. Villard, in Journal of Physics: Conference Series, Vol. 561 (IOP Publishing, 2014) p. 012017.

[25] J. Chowdhury, Y. Chen, W. Wan, S. E. Parker, W. Guttenfelder, and J. Canik, Phys. Plasmas 23, 012513 (2016).

[26] Y. Chen, J. Chowdhury, N. Maksimovic, S. Parker, and W. Wan, Phys. Plasmas 23, 056101 (2016).

[27] J. Chowdhury, Y. Chen, and S. E. Parker, Physics of Plasmas 27, 042309 (2020).

[28] W. Tang, J. Connor, and R. Hastie, Nucl. Fusion 20, 1439 (1980).

[29] M. Coury, W. Guttenfelder, D. Mikkelsen, J. Canik, G. Canal, A. Diallo, S. Kaye, G. Kramer, R. Maingi, and N.-U. team, Phys. Plasmas 23, 062520 (2016).

[30] G. L. Falchetto, J. Vaclavik, and L. Villard, Phys. Plasmas 10, 1424 (2003).

[31] R. Ganesh, P. Angelino, J. Vaclavik, and L. Villard, Phys. Plasmas 11, 3106 (2004).

[32] Y.-K. Pu and S. Migliuolo, Phys. fluids 28, 1722 (1985).

[33] A. Smolyakov, M. Yagi, and Y. Kishimoto, Phys. Rev. Lett. 89, 125005 (2002).

[34] A. Hirose, M. Elia, A. Smolyakov, and M. Yagi, Phys. Plasmas 9, 1659 (2002).

[35] Z. Gao, H. Sanuki, K. Itoh, and J. Dong, Phys. Plasmas 10, 2831 (2003).

[36] Z. Gao, J. Dong, and H. Sanuki, Phys. Plasmas 11, 3053 (2004).

[37] Z. Gao, H. Sanuki, K. Itoh, and J. Dong, Phys. Plasmas 12, 022502 (2005).

[38] Z. Gao, H. Sanuki, K. Itoh, and J. Dong, J. Plasma Phys. 72, 1249 (2006).

[39] J. Chowdhury, R. Ganesh, J. Vaclavik, S. Brunner, L. Villard, and P. Angelino, Phys. Plasmas 16, 082511 (2009).

[40] J. Chowdhury, S. Brunner, R. Ganesh, X. Lapillonne, L. Villard, and F. Jenko, Phys. Plasmas 19, 102508 (2012).

[41] M. Han, Z.-X. Wang, J. Dong, and H. Du, Nucl. Fusion 57, 046019 (2017).

[42] F. Wagner, G. Becker, K. Behringer, D. Campbell, A. Eberhagen, W. Engelhardt, G. Fussmann, O. Gehre, J. Gernhardt, G. v. Gierke, et al., Phys. Rev. Lett. 49, 1408 (1982).

[43] G. Merlo, S. Brunner, Z. Huang, S. Coda, T. Goerler, L. Villard, A. B. Navarro, J. Dominski, M. Fontana, F. Jenko, et al., Plasma Phys. Control. Fusion 60, 034003 (2018). 
[44] J. Citrin, J. Garcia, T. Görler, F. Jenko, P. Mantica, D. Told, C. Bourdelle, D. Hatch, G. Hogeweij, T. Johnson, et al., Plasma Phys. Control. Fusion 57, 014032 (2014).

[45] J. Weiland and A. Hirose, Nucl. Fusion 32, 151 (1992).

[46] A. Hirose, Phys. Plasmas 7, 433 (2000).

[47] S. Liu, W. Kong, B. Hu, F. Liu, J. Dong, and Z. Gao, Phys. Plasmas 16, 012302 (2009).

[48] J. Kim, W. Horton, and J. Dong, Phys. Fluids B: Plasma Physics 5, 4030 (1993).

[49] G. L. Falchetto, Electromagnetic microinstabilities in tokamak plasmas using a global spectral approach, Ph.D. thesis, EPFL, Lausanne (2002).

[50] R. Ganesh and J. Vaclavik, Phys. Rev. Lett. 94, 145002 (2005).

[51] S. Brunner and J. Vaclavik, Phys. Plasmas 5, 365 (1998).

[52] S. Brunner, M. Fivaz, T. Tran, and J. Vaclavik, Phys. Plasmas 5, 3929 (1998).

[53] S. Brunner, Global approach to the spectral problem of microinstabilities in tokamak plasmas using a gyrokinetic model, Ph.D. thesis, EPFL, Lausanne (1997).

[54] A. K. Swamy, R. Ganesh, J. Chowdhury, S. Brunner, J. Vaclavik, and L. Villard, Phys. Plasmas 21, 082513 (2014).

[55] J. Weiland, Collective Modes in Inhomogeneous Plasmas: Kinetic and Advanced Fluid Theory, Series in Plasma Physics and Fluid Dynamics (Taylor \& Francis, 1999). 